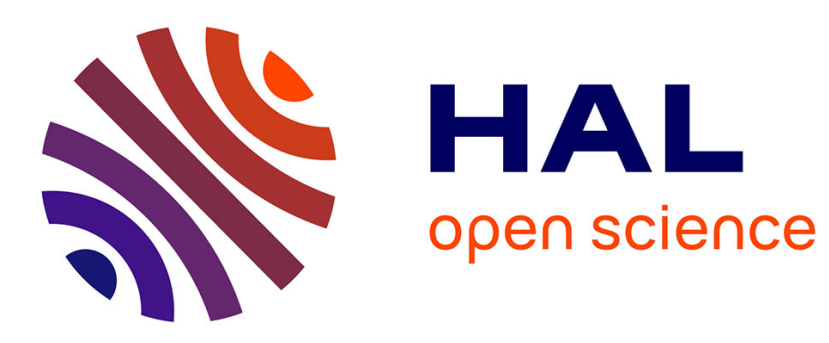

\title{
Understanding and modeling the small-world phenomenon in dynamic networks
}

Anh-Dung Nguyen, Patrick Sénac, Michel Diaz

\section{To cite this version:}

Anh-Dung Nguyen, Patrick Sénac, Michel Diaz. Understanding and modeling the small-world phenomenon in dynamic networks. The 15th ACM International Conference on Modeling, Analysis and Simulation of Wireless and Mobile Systems (MSWIM 2012), Oct 2012, Paphos, Cyprus. pp.377-384. hal-00908491

\section{HAL Id: hal-00908491 \\ https://hal.science/hal-00908491}

Submitted on 23 Nov 2013

HAL is a multi-disciplinary open access archive for the deposit and dissemination of scientific research documents, whether they are published or not. The documents may come from teaching and research institutions in France or abroad, or from public or private research centers.
L'archive ouverte pluridisciplinaire HAL, est destinée au dépôt et à la diffusion de documents scientifiques de niveau recherche, publiés ou non, émanant des établissements d'enseignement et de recherche français ou étrangers, des laboratoires publics ou privés. 


\section{Understanding and Modeling the Small-World Phenomenon in Dynamic Networks}

\author{
Anh-Dung Nguyen \\ ISAE-University of Toulouse \\ LAAS-CNRS \\ Toulouse, France \\ anh- \\ dung.nguyen@isae.fr
}

\author{
Patrick Senac \\ ISAE-University of Toulouse \\ LAAS-CNRS \\ Toulouse, France \\ patrick.senac@isae.fr
}

\author{
Michel Diaz \\ LAAS-CNRS \\ Toulouse, France \\ michel.diaz@laas.fr
}

\begin{abstract}
The small-world phenomenon first introduced in the context of static graphs consists of graphs with high clustering coefficient and low shortest path length. This is an intrinsic property of many real complex static networks. Recent research has shown that this structure is also observable in dynamic networks but how it emerges remains an open problem. In this paper, we propose a model capable of capturing the small-world behavior observed in various real traces. We then study information diffusion in such smallworld networks. Analytical and simulation results with epidemic model show that the small-world structure increases dramatically the information spreading speed in dynamic networks.
\end{abstract}

\section{Categories and Subject Descriptors}

G.2.2 [Graph Theory]: Network problems; C.2.1 [Network Architecture and Design]: Store and forward networks

\section{General Terms}

Theory, Measurement

\section{Keywords}

Small-world, dynamic networks, information diffusion

\section{INTRODUCTION}

In his famous experiment Milgram showed that the human acquaintance network has a diameter of the order of six, so leading to the small-world qualification. Watts and Strogatz introduced later a model of small-world phenomenon for static graphs 10. They proposed a random rewiring procedure for interpolating between a regular ring lattice and a random graph. Between these two extrema, the graph exhibits an exponential decay of the average shortest path

Permission to make digital or hard copies of all or part of this work for personal or classroom use is granted without fee provided that copies are not made or distributed for profit or commercial advantage and that copies bear this notice and the full citation on the first page. To copy otherwise, to republish, to post on servers or to redistribute to lists, requires prior specific permission and/or a fee.

Copyright 20XX ACM X-XXXXX-XX-X/XX/XX ...\$10.00. length contrasted to a slow decay of the average clustering coefficient. Interestingly, numerous real static networks exhibit such properties.

From a communication perspective, it was shown that a small-world network behaves as random network in terms of information diffusion. However, the great majority of studies on small-world networks properties and behaviors focused on static graphs and ignore the dynamics of real mobile networks. For example, in a static graph an epidemic cannot break out if the initial infected node is in a disconnected component of the network; conversely in a mobile network, nodes movements can ensure a property of temporal connectivity of the underlying dynamic graph. Moreover, an epidemic can take off or die out depending not only on the network structure and the initial carrier, but also on the time when the disease begins to spread. These aspects cannot be captured by a static small-world model.

In this paper, we address this issue by introducing a model for dynamic small-world networks. In particular, this model aims to capture the small-world phenomenon that emerges from delay/disruption tolerant networks. Starting from a highly clustered dynamic network where nodes tend to stay all the time in their preferential zones, we introduce a dynamic rewiring procedure which consist in progressively increasing the ratio of nomadic nodes. This allows to cover a whole scope of dynamic networks from highly clustered ones to fully random ones.

In order to formalize the dynamic small-world phenomenon, we extend to dynamics networks the notions of dynamic clustering coefficient and shortest dynamic path. By studying the evolution of these metrics from our rewiring process, we exhibit the emergence of a class of dynamic small-world networks with high dynamic clustering coefficients and low shortest dynamic path length. This simulation-based work is then extended by an analytical study of information spreading in dynamic small-world networks.

The rest of the paper is structured as follows. Section 2 introduces previous works that inspired our contribution. In Section 3 after having formally defined the notions of dynamic clustering coefficient and shortest dynamic path length, we analyze various real traces and show the intrinsic properties of dynamic networks that induce the smallworld phenomenon. We introduce then a dynamic smallworld model which captures these properties in Section 4 In Section 5 from simulations results and analytical formulation we study information diffusion performances in dynamic 
small-world networks. Finally, Section 6 will conclude the paper and discuss about future works.

\section{RELATED WORKS}

In 10, Watts and Strogatz introduce a model of smallworld phenomenon in static graphs. From a regular ring lattice, they rewire randomly edges in this graph with a probability varying from 0 (i.e. leading to a regular network) to 1 (i.e. leading a random graph). During this process they observed an abrupt decrease of the average shortest path length, leading to short path of the same order of magnitude as observed in random graphs, while the clustering coefficient is still of the same order of magnitude than the one of a regular graph. This features suggested the emergence of the small-world phenomenon. The authors also demonstrate that this graph structure allows information to diffuse as fast as in a random graph.

Kleinberg 3 extended the model to 2-d lattices and introduced a new rewiring process. This time, the edges are not uniformly rewired but follow a power law $\frac{1}{d^{\alpha}}$ where $d$ is the distance on the lattice from the starting node of the edge and $\alpha$ is the parameter of the model. Newman [5] proposed another definition of the clustering coefficient which has a simple interpretation and is easier to process. The authors argue that the definition in 10 favors vertices with low degree and introduces a bias towards networks with a significant number of such vertices.

Although research pays much attention to the small-world phenomenon in static networks, whether through modeling or analytical analysis, the small-world phenomenon in dynamic networks (i.e. networks with time varying edges and nodes) is still not well understood. This is partly due to the lack of models and metrics for dynamic graphs. Recently, J.Tang et al. 8 defined several metrics for time varying graphs, including temporal path length, temporal clustering coefficient and temporal efficiency. They showed that these metrics are useful to capture temporal characteristics of dynamic networks that cannot be captured by traditional static graph metrics. The definition of dynamic path, introduced in this paper, is close to their definition (we had the dual metric of the number of hops). We introduce a new definition of dynamic clustering coefficient which, as shown in the next section, captures more accurately the dynamics of networks.

In 9, the authors highlight the existence of the smallworld behavior in real traces. Using the definition of temporal correlation introduced in 2, they show that real dynamic networks have a high temporal correlation and low temporal shortest path, suggesting a dynamic small-world structure. In this paper, we simply and consistently extend to dynamic networks the initial small-world metrics (i.e. shortest path length and clustering coefficient) defined in 10. Finally, the analytical study of information diffusion in dynamic smallworld introduced in this paper is inspired from the method applied for static networks described by Newman in 6.

\section{SMALL-WORLD PHENOMENON IN DY- NAMIC NETWORKS}

In this section, we formalize the notion of small-world phenomenon in dynamic networks by introducing two metrics used for qualifying such phenomenon: shortest dynamic path length and dynamic clustering coefficient. And then by analyzing extensively real network traces, we show fundamental characteristics which are at the origin of the dynamic small-world phenomenon.

\subsection{Dynamic Small-world Metrics}

\subsubsection{Shortest Dynamic Path Length}

Basically, the shortest path problem in static graphs consists in finding a path such that the sum of the weights of its constituent links is minimized. From a graph theory point of view a dynamic networks can be described by a temporal graph, that is a temporal sequence of graphs that describe the discrete evolution of the network according to nodes and links creation and destruction events. A path in a dynamic network can be seen as an ordered set of temporal links that allow a message to be transferred using the store-moveforward paradigm between two nodes. Formally, let $l_{i j}^{t}$ be a link between node $i$ and node $j$ at instant $t$. A dynamic path from node $u$ to node $v$ from time $t_{0}$ to time $t$ is described by a time ordered set $p_{u v}\left(t_{0}, t\right)=\left\{l_{u i}^{t_{0}}, l_{i j}^{t_{1}}, \ldots, l_{w v}^{t}\right\}$ where $t_{k+1}>t_{k}$. We consider two metrics of dynamic paths:

- Delay : the sum of the inter-contact times between consecutive links which constitutes the path.

- Number of hops : the number of temporal links which constitutes the path.

This leads to the following definition of shortest dynamic path length.

Definition 1. The shortest dynamic path is the path giving the minimum amount of delay ${ }^{1}$ If there are several paths giving the same delay, then we select the one giving the minimum number of hops. Formally, the shortest dynamic path length between $i$ and $j$ from time $t_{0}$ is

$$
\mathcal{L}_{i j}^{t_{0}}=\inf \left\{t-t_{0} \mid \exists p_{i j}\left(t_{0}, t\right)\right\} .
$$

The shortest dynamic path length of a network of $N$ nodes from time $t_{0}$ is the average of the shortest dynamic path lengths of all pairs of nodes in the network

$$
\mathcal{L}^{t_{0}}=\frac{\sum_{i j} \mathcal{L}_{i j}^{t_{0}}}{N(N-1)} .
$$

To find the shortest dynamic path length of all pairs of nodes, we propose an algorithm leveraging on the following interesting property of adjacency matrix in static graphs.

The adjacency matrix $A$ is defined as the matrix in which the element $(A)_{i, j} \in\{0,1\}$ at row $i$ and column $j$ denotes the existence of a link between node $i$ and node $j$. If we process the power $n$ of such matrix, then its element $\left(A^{n}\right)_{i, j}$ gives the number of paths of length $n$ between $i$ and $j$. Indeed, for example, when $n=2,\left(A^{2}\right)_{i, j}=\sum_{k}(A)_{i, k} \times(A)_{k, j}$ sums all the possibilities to go from $i$ to $j$ through an intermediate node $k$. We extend this property to dynamic networks. Let $A_{t}, t=0,1, \ldots, n$ be the adjacency matrix of a dynamic network at time $t$. The matrix $C_{t}$ obtained as follows

$$
C_{t}=\mathbf{A}_{t} \vee \mathbf{A}_{t}^{2} \vee \ldots \vee \mathbf{A}_{t}^{n}
$$

where $\mathbf{A}_{t}^{i}$ denotes the binary version of the matrix $A_{t}^{i}$ (i.e. the element $\left(\mathbf{A}_{t}\right)_{i, j}$ equals to 1 if $\left(A_{t}\right)_{i, j}>0$ and 0 otherwise)

${ }^{1}$ we can also have another definition for minimizing the number of hops. In this work, we focus only on delay constrained path. 
has its elements $\left(C_{t}\right)_{i, j}$ which indicate if there is a direct or indirect link (up to $n$ hops) between $i$ and $j$ at time $t$. Indeed, $\left(C_{t}\right)_{i, j}$ is the logical sum of all possibilities to have a direct or indirect link (up to $n$ hops) between $i$ and $j$ at time $t$. In consequence, the product

$$
D_{t}=C_{0} C_{1} \ldots C_{t}
$$

results in a matrix in which the element $\left(D_{t}\right)_{i, j}$ specifies, when not null, that there is dynamic path of delay $t$ between $i$ and $j$. Therefore the shortest dynamic path length from node $i$ to node $j$ is given by the smallest value of $t$ such as $\left(D_{t}\right)_{i, j}$ equals to 1 .

It is straight-forward to demonstrate that if a node $k$ belongs to a shortest path between node $i$ and node $j$ then the $i$ to $k$ sub-path gives the shortest path between $i$ and $k$. Therefore the shortest path between two nodes $i$ and $j$ can be easily backwardly reconstructed. Note that at time $t$ two nodes can be connected to each other via a multiple hops link. To find the complete spatio-temporal path with all the intermediate nodes, we simply apply a breath first search each time we find a spatial multiple hops link. In practice, as it's unlikely to have a large number of nodes connected to each other at a given moment, we can optimize the algorithm by limiting the number of iterations $n$ in Equation 3 to an upper bound of the network diameter. Finally, this algorithm is more efficient (time complexity $O\left(n^{3}\right)$ ) than the depth first search approach as proposed in 8. (time complexity $O\left(n^{4}\right)$ ).

\subsubsection{Dynamic Clustering Coefficient}

As defined in [10, the clustering coefficient measures the cliquishness of a typical friend circle. The clustering coefficient of a node is calculated as the fraction of actual existing links between its neighbors and the number of possible links between them. The clustering coefficient of a network is calculated by averaging the clustering coefficients of all the nodes in the network. In 5], Newman defines the clustering coefficient in term of transitivity. The connection between nodes $u, v, w$ is said to be transitive if $u$ connected to $v$ and $v$ connected to $w$ implies that $u$ is connected to $w$. The clustering coefficient of a network is then calculated as the fraction of the number of closed path of length two over the number of path of length two, where a path of length two is said to be closed if it is a transitive path. This definition is simple to interpret and easy to calculate. Considering that, the initial definition gives more weight to nodes with low degree and introduces a bias towards graph composed of several of these nodes, in this work, we favor Newman's definition and extend it to dynamic networks.

In 8, Tang et al. first introduced a generalization of Watts and Strogatz's definition for temporal graph. The temporal clustering coefficient of a node during a time interval $t$ is the fraction of existing contacts ${ }^{2}$ (multiple contacts count once) between the neighbors of the node over the number of possible contacts between them during $t$. The clustering coefficient of the network is the average of the clustering coefficients of all the nodes. This is simply the application of Watts and Strogatz's definition on a time snapshot of the network. While this definition was shown to better capture temporal characteristics of time varying graph, it depends strongly on the larger of the chosen time interval. If this

\footnotetext{
${ }^{2} \mathrm{~A}$ contact is a intermittent link between two nodes
}

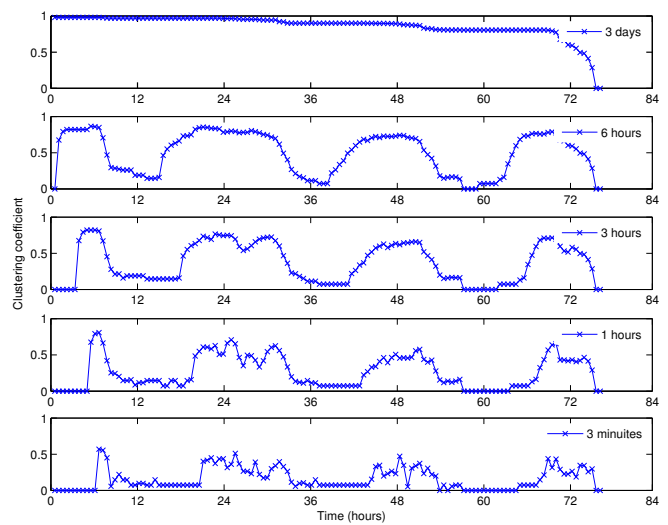

Figure 1: Time window size effect on measure of temporal clustering coefficient (as defined in [11])

\begin{tabular}{ccccc}
\hline Trace & Intel & Cambridge & Infocom05 & Infocom06 \\
\hline Number of nodes & 9 & 12 & 41 & 78 \\
Duration (days) & 6 & 6 & 4 & 4 \\
Granularity (seconds) & 120 & 120 & 120 & 120 \\
Connectivity & Bluetooth & Bluetooth & Bluetooth & Bluetooth \\
Environment & Office & Office & Conference Conference \\
\hline
\end{tabular}

Table 1: Data sets of real traces

interval tends to infinity, the temporal clustering coefficient tends to 1 as all the nodes meet each other with a high probability. On the other hand, if the interval tends to 0 , then conversely the temporal clustering coefficient tends to 0 . Figure 1 illustrates the influence of the time window size on measures of the clustering coefficient on a real mobility trace.

In order to avoid this temporal bias, we propose a new definition of dynamic clustering coefficient which captures the dynamics of the degree of transitivity and is independent of the measuring time interval.

Definition 2. A dynamic path from node $i$ to node $j$ is transitive if there exists a node $k$ and time $t_{1}, t_{2}, t_{3}$ so that $i$ is connected to $k$ at $t_{1}, k$ is connected to $j$ at $t_{2}, i$ is connected to $j$ at $t_{3}$ and $t_{1} \leq t_{2} \leq t_{3}$. The dynamic clustering coefficient of node $i$ from time $t_{0}$ is measured by the inverse of the time $t-t_{0}$ where $t$ is the first instant from time $t_{0}$ when the transitive path from $i$ to $j$ is formed, that is $\mathcal{C}_{i}^{t_{0}}=\frac{1}{t-t_{0}}$. The dynamic clustering coefficient of a network of $N$ nodes is then calculated by averaging over the dynamic clustering coefficient of all the nodes from time $t_{0}$

$$
\mathcal{C}=\frac{1}{N} \sum_{i} \mathcal{C}_{i}
$$

\subsection{Network Traces Analysis}

In this section, we analyze extensively real human connectivity traces to understand how small-world behavior emerges in dynamic networks. We first apply the above definitions to highlight the existence of dynamic small-world phenomenon on these traces. For that, we use the data sets from the Haggle project 1, 4. which consists of the recording of opportunistic bluetooth contacts between users in conference or office environments. The settings of these data sets are summarized in Table 1. 


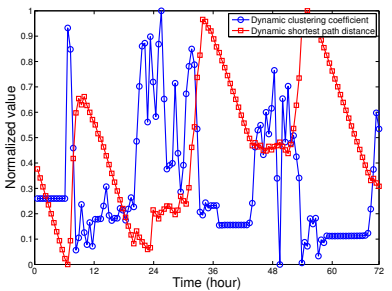

(a) Infocom 2005

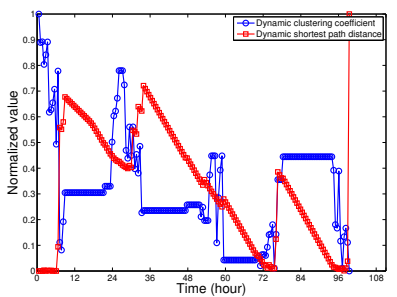

(c) Intel

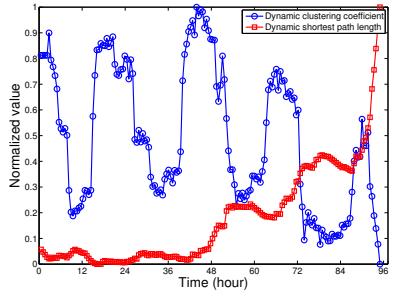

(b) Infocom 2006

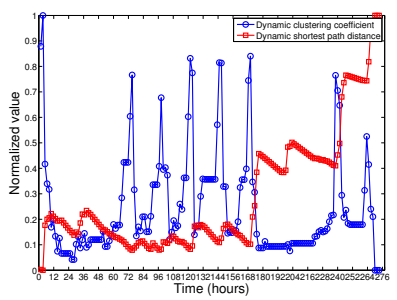

(d) Cambridge
Figure 2: Small world phenomenon observed in real mobility traces

For each trace, we measure the shortest dynamic path length and the dynamic clustering coefficient every 2000 seconds to see the evolution of these metrics over time. The obtained values are then normalized and plotted on Figure 2 We can observe a periodic pattern of both metrics with a typical period of 24 hours and a phase change every 12 hours. This can be easily explained by the fact that human daily activity is periodic with nigh/day phases. Indeed, people are more nomadic during day while they are mostly sedentary at night. Besides, it is interesting to note that the dynamic clustering coefficient and the shortest dynamic path length evolve in opposite phase. Despite of a slight diversity in different traces due to differences in nodes number and density, traces durations, etc, during the dynamic phase (i.e., day, e.g., the period around 24 hours in Infocom2005 trace), these networks always exhibit high dynamic clustering coefficients and low shortest dynamic path lengths, suggesting the existence of a dynamic small-world phenomenon.

To explain the emergence of this phenomenon, let us focus on and analyze the structure of these networks during the dynamic phases. In the Watts and Strogatz model, the small-world phenomenon emerges when shortcut edges are randomly added to a regular graph. These shortcuts allow the average shortest path length to be reduced significantly while conserving network nodes's cliquishness. We argue that in dynamic networks, mobile nodes are implicitly at the origin of these shortcuts. Indeed, it is known that people spend their daily life among different social communities at specific locations at different times (e.g. colleagues at office in the morning, family at home in the evening). A community can be disconnected from the others in space and/or in time. Besides, some people are more "mobile" than other, they have contacts in many communities and move often between these communities or areas. These "nomadic" nodes contribute to reducing significantly the shortest dynamic path length from nodes in a disconnected component to the rest of network and hence contribute to the emergence of the dynamic small-world phenomenon.

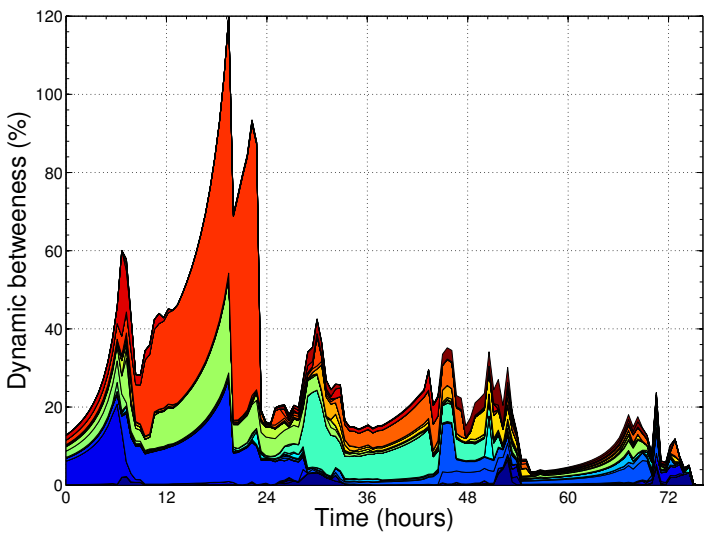

Figure 3: Evolution of dynamic betweeness centrality of nodes in Infocom05 trace

To identify the spatio-temporal shortcuts in dynamic networks, we introduce a metric that measures the influence a node has on the characteristic dynamic path length of the network. The nodes with highest influence are the ones whose removal from the network increases the most the average shortest dynamic path length of the network. To identify these nodes one may adapt to dynamic networks the notion of betweeness centrality already introduced for static networks. In the context of dynamic networks, we call it dynamic betweeness centrality. Consider a node $i$, first of all we measure the average of the shortest dynamic path length between all pairs of nodes $s, t$ except paths from and to $i$. Then, we remove $i$ from the network and perform the same measure. The dynamic betweeness centrality of $i$ is defined as the ratio between these two measures. Formally, that is

$$
x_{i}=\frac{\sum_{s t} \mathcal{L}^{\prime}{ }_{s t}}{\sum_{s t} \mathcal{L}_{s t}},
$$

where $\mathcal{L}^{\prime}{ }_{s t}$ and $\mathcal{L}_{s t}$ are respectively the shortest dynamic path lengths from $s$ to $t$ after and before removing $i$.

We measure the dynamic betweeness of all nodes at different times on the traces. Figure 3 shows the temporal evolution of the dynamic betweeness for the Infocom05 trace. On the figure, the different areas represent the measured metric (in \%) of different nodes. We can observe that the dynamic betweeness of a nodes changes over time and that there are some nodes with very high influence whose removal would dramatically increase the dynamic characteristic path length of the network. Indeed, at the time of the highest pick, the most influential node decreases of almost $70 \%$ the average shortest dynamic path length of the network. We can also see the abrupt decrease of the average shortest dynamic path length at time 24 hours on Figure 2(a) The time average of the dynamic betweeness centrality of each network node allows one to rank the influence of each node during a time window. For instance in Figure 3 the highest value for the daytime period corresponds to node with ID 34.

Now let us look at the highest pick to see what happened in the network at that moment. We measure the impact of node 34 on the shortest dynamic paths from and to each other node (respectively denoted out/in shortest dynamic path on Figure 4. Figure 4 shows that node 34 has very high influence on node 31 . We then apply our all-pairs- 


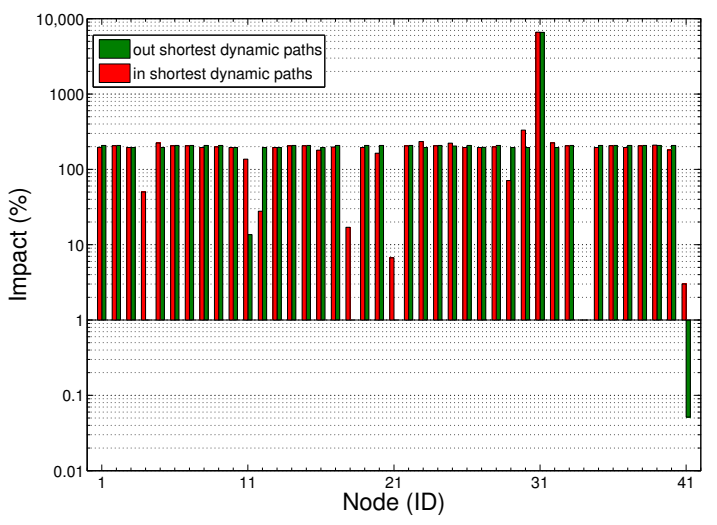

Figure 4: Impact of node 34 on the shortest dynamic paths from and to the other nodes at $t=22 \mathrm{~h}$ (Infocom05 trace)

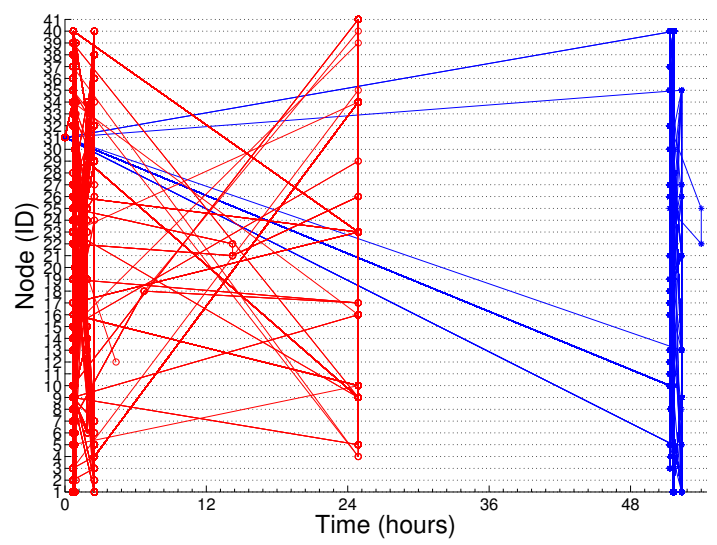

Figure 5: Shortest dynamic paths from node 31 at $t=22 \mathrm{~h}$ in Infocom05 trace, before (in red) and after (in blue) removal of node 34

shortest-dynamic-path algorithm to find all the shortest dynamic paths from node 31 to others before and after removing node 34 . Figure 5 shows that node 34 is crucial for node 31 to efficiently communicate with other nodes at that instant. Therefore node 34 plays the role of a spatio-temporal shortcut between node 31 and the other nodes. This result can be observed for any node with a high dynamic betweeness. Indeed, if the so disconnected network part is a giant component, then the impact of the removal of a dynamic shortcut can dramatically increase the characteristic dynamic path length of the network.

\section{DYNAMIC SMALL-WORLD MODELING}

The previous section showed that the dynamic work small world phenomenon is intrinsic to a great diversity of dynamic network traces. Therefore, the definition of a model that abstracts this phenomenon in order to study the impact of its emergence on dynamic networks communications performances is a significant issue. In this section, we introduce a model for dynamic small-worlds and show its modeling and expressive power. As evoked in the introduction, this model aims to capture the dynamic small-world phenomenon resulting from human mobility behavior through human char- acteristics described in the previous section. That is:

- People move between clusters or communities

- Some people are nomad while the others are more sedentary. These nomadic nodes move from cluster to cluster and hence contribute to reducing the network diameter.

\subsection{The model}

Consider a mobile network in which each node belongs to a preferential attachment zone or clusters. We introduce two types of nodes: sedentary nodes which move only inside their preferential zon ${ }^{3}$ and nomadic nodes which can move between zones. Nodes moving in two different zones cannot be connected and therefore nodes in different zones can communicate only through the movement of nomadic nodes. Initially, nomadic nodes are uniformly distributed over all clusters. The fraction of nomadic nodes is $p$ which is a parameter of the model.

Obviously, when $p$ equals to 0 , there is no possible communication between nodes in different zones and hence the network is totally partitioned in disconnected zones. On the contrary, when $p$ equals to 1 , all the nodes are nomadic and hence there are dynamic paths between clusters and in consequence the network is highly connected. In consequence, the rewiring process consist in varying $p$ from 0 to 1 . We are interested in the properties of networks which are formed between these two extrema.

As we have demonstrated in a previous work 7], human mobility can be modeled with STEPS - a simple model implementing preferential attachment and attractor via a power law. We apply this mobility model to the movement of nomadic nodes. The node chooses the zone to move in with a probability inversely proportional to the distance between his preferential zone and the chosen zone. That is

$$
P\left(Z=z_{i}\right)=\frac{\lambda}{\left(1+d_{z_{0} z_{i}}\right)^{\alpha}},
$$

where $z_{0}$ is the preferential cluster of the node, $\lambda$ is constant of normalization and the power law exponent $\alpha$ a parameter of STEPS model.

\subsection{Simulation Results}

We simulate in MATLAB a mobile network of 1000 mobile nodes. The simulated network area of size $200 \times 200 \mathrm{~m}^{2} \mathrm{di}-$ vided into $20 \times 20$ zones as shown in Figure 6 gives a density of 25000 nodes $/ \mathrm{km}^{2}$ which corresponds to the population density of a large city like Paris. We distribute uniformly nodes over zones and set the radio range to $10 \mathrm{~m}$ which corresponds to bluetooth technology. While varying the fraction of nomadic nodes from 0 to 1 , we measure the dynamic clustering coefficient and shortest dynamic path length of resulting networks.

Figure 7 plots the resulting average over 10 simulations with the corresponding $95 \%$ confidence intervals. We observe that the shortest dynamic path length drops rapidly as soon as we introduce a small percentage of nomadic nodes (i.e. less than $10 \%$ ) into the network whilst the clustering coefficient remains very high. This result obtained from a simple model of evolution of a dynamic network demonstrates, identically to the simple model used to exhibit the

${ }^{3}$ following a random mobility model such as Random Waypoint 


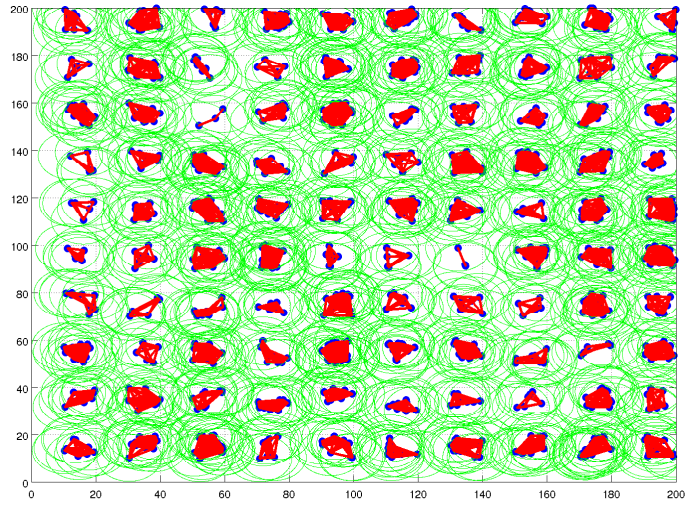

Figure 6: Small-world network configuration

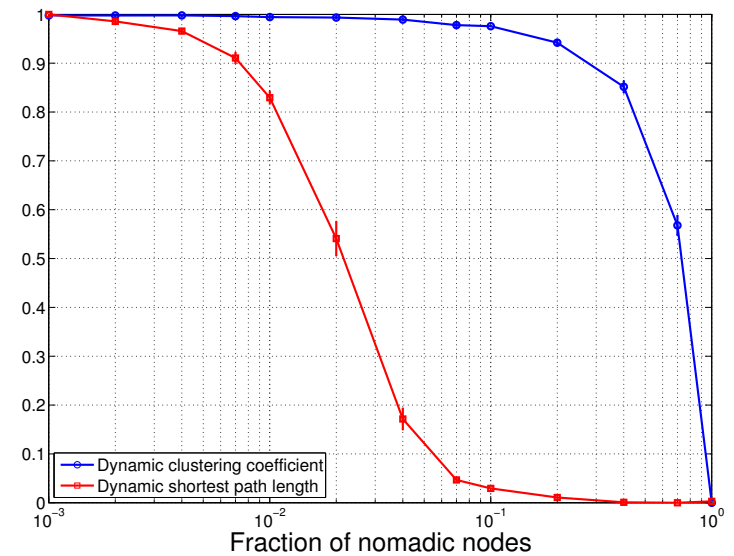

Figure 7: Small-world phenomenon in dynamic networks

emergence of the small world phenomenon in static networks 10, how the small-world phenomenon emerges from dynamic networks.

\section{INFORMATION DIFFUSION IN DYNAMIC SMALL-WORLD NETWORKS}

Following the understanding and modeling of the small world phenomenon in dynamic networks, this section aims to study the effect of the dynamic small-world phenomenon on information diffusion performances. We present analytical and simulation results obtained with the Susceptible Infected (SI) epidemic model. We apply SI model on a simplified version of the dynamic small-world model used in the previous section in which we make abstraction of nodes displacement inside sites. Nodes movements are limited to jumping from one site to another. Simulation results for the complete version of the model are also provided. Let us consider a square lattice of $N$ sites with initially 1 node in each site, node $i$ is associated to site number $i$. Assume that when 2 nodes are in the same site, they are connected. At each time $t$, mobile nodes jump to another site while sedentary nodes stay where they are. Initially, we suppose 1 infected node and $100 \%$ of other nodes susceptible. We for- mulate the dynamics of the infection of this network through a differential equation as follows.

Consider a node $i$. Let $x_{i}(t)$ be the random variable which denotes the probability of node $i$ being infected at time $t$. Let us express the probability that $i$ becomes infected between time $t$ and $t+d t$. A static node $i$ can be infected by a mobile node $m$ only. This happens with the probability $\delta_{m i}$ that node $m$ jumps to site $i$. The probability that the infection (i.e. information) is transmitted during the interval $d t$ is $\beta d t$ where $\beta$ is the transmission rate - a standard parameter of the SI epidemic model. Summing over all the mobile nodes and then multiplying by the probability $1-x_{i}$ that $i$ is not infected at time $t$, we obtain the differential equation

$$
\frac{d x_{i}}{d t}=\beta\left(1-x_{i}\right) \sum_{m} \delta_{m i} x_{m} .
$$

Conversely, for a mobile node, $i$, to be infected, it must be in contact with a mobile or static node already infected. If a node $i$ jumps to a site $j$ where resides an static node, this happens with probability $x_{j}+\sum_{m \neq i} \delta(m i) x_{m}$ and hence

$$
\frac{d x_{i}}{d t}=\beta\left(1-x_{i}\right)\left(x_{j}+\sum_{m \neq i} \delta_{m j} x_{m}\right) .
$$

If the destination site $j$ is associated to a mobile node then this probability is $\sum_{m \neq i} \delta_{m j} x_{m}$ and hence

$$
\frac{d x_{i}}{d t}=\beta\left(1-x_{i}\right) \sum_{m \neq i} \delta_{m j} x_{m} .
$$

Combining all these cases, we obtain the following matrix differential equation describing the dynamics of the system

$$
\begin{aligned}
\frac{d \mathbf{x}}{d t}= & \beta(1-\mathbf{x}) \circ\langle(1-m) \circ[\mathbf{D}(m \circ \mathbf{x})]+ \\
& +m \circ\{\mathbf{D}[(1-m) \circ \mathbf{x}+\mathbf{D}(m \circ \mathbf{x})]\}\rangle,
\end{aligned}
$$

where $\mathbf{x}=\left[x_{1} \ldots x_{N}\right]^{\prime}$ is the random vector of nodes infection probability and $m$ is the binary vector in which $m_{i}=1$ for a mobile node and $m_{i}=0$ for a static node. The matrix

$$
\mathbf{D}=\left[\begin{array}{ccc}
\delta_{11} & \ldots & \delta_{1 N} \\
\vdots & \ddots & \vdots \\
\delta_{N 1} & \ldots & \delta_{N N}
\end{array}\right]=\lambda\left[\begin{array}{ccc}
1 & \cdots & \frac{1}{\left(1+d_{1 N}\right)^{\alpha}} \\
\vdots & \ddots & \vdots \\
\frac{1}{\left(1+d_{N 1}\right)^{\alpha}} & \ldots & 1
\end{array}\right]
$$

is the stochastic matrix describing the stationary state of the system.

To have an approximated solution of Equation 11, we integrate it by numerical method. The results are shown in Figure 8 which plots the time evolution of the fraction of infected nodes for a 10000 nodes network. The four curves correspond a fraction of mobile nodes $p_{m}$ equals to $0.1 \%, 1 \%, 10 \%, 100 \%$ respectively. The coefficient $\alpha$ for mobile node is equal to 0 and the transmission rate $\beta$ is equal to 1 . It is interesting to see that for a low fraction of mobile nodes, epidemic spreads very slowly because this fraction is still below the percolation threshold of the network, while with only $10 \%$ (i.e. as soon as the small-world structure emerges) the epidemic breaks out rapidly. We also ran simulations with a network of 100 nodes, $\alpha$ equals to $0, \beta$ equals to 1 and different values for the fraction of mobile nodes. The average over 100 simulations is then compared with the corresponding analytical results. Figure 9 shows 


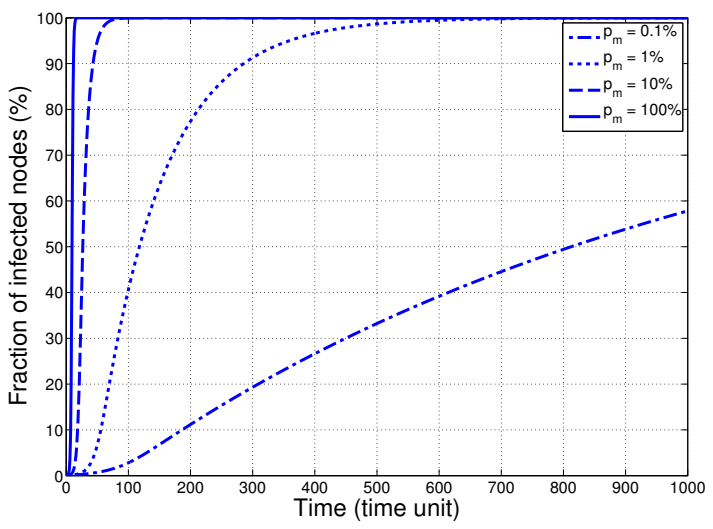

Figure 8: Evolution of fraction of infected nodes over time (theory)

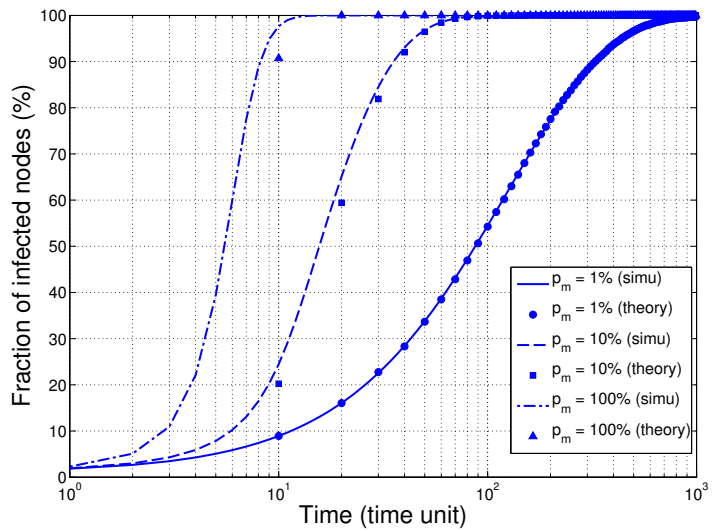

Figure 9: Comparison of analytical and simulation results

the strong accuracy and compliance of our analytical model with the simulation results. Finally, we run simulation with the complete version of the small-world model. The result is the average over 10 simulations of a 100 nodes network with the same configurations. Figure 10 shows a similar result compared to the first case. The effect of displacement time between zones only spreads the curves so that the epidemic take much longer time to take off.

\section{CONCLUSION}

In this paper, we first proposed a model that explains the formation, evolution, behavior and performances of dynamic small-world networks. In this model, from a clusterized network composed of nodes with localized moves, we perform a rewiring process which consists in introducing nomadic nodes into the network. These nodes play the role of bridges between disconnected clusters and reduce significantly the dynamic shortest paths between nodes in the network. From the study of real mobility traces we showed that our model expresses salient properties observed in real dynamic networks. Finally, based on this model and from a joint use of analytical analysis and simulations, we have studied information diffusion in dynamic small-world networks, for the SI epidemic model. We showed that the emergence of a dynamic small-world structure in dynamic networks is accom-

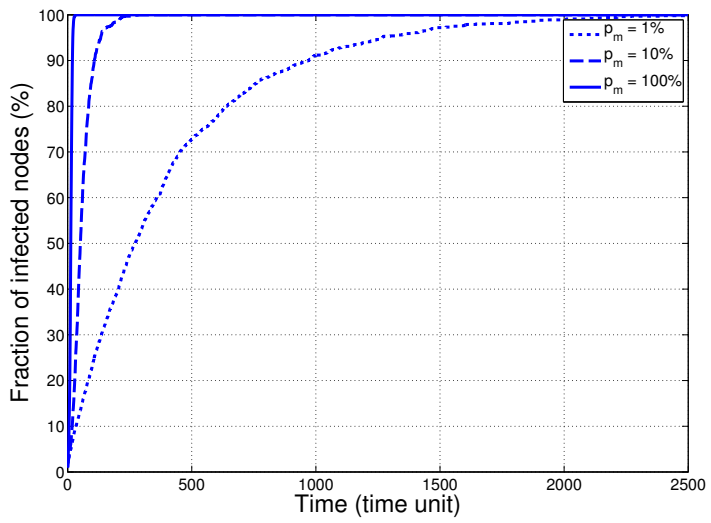

Figure 10: Evolution of fraction of infected nodes over time with the complete version of the model (simulation result)

panied by a sudden improvement of information diffusion performances in the network. Our on-going work aims to address this issue for more complex epidemic models such as SIR or SIRS. A study of the percolation threshold and percolation process in dynamic small-world networks is also an significant problem to be considered. Although in this paper, one contributing factor was found, further unique properties of dynamic networks might be contributing as well and hence should also be considered. For our best knowledge, this is the first model of small-world phenomenon in dynamic networks. This model could be integrated in existing dynamic network simulators (e.g. theONE) to evaluate protocol performances (at network or transport level) in such type of network.

\section{REFERENCES}

[1] Augustin Chaintreau, Pan Hui, Jon Crowcroft, Christophe Diot, Richard Gass, and James Scott. Impact of human mobility on opportunistic forwarding algorithms. IEEE Transactions on Mobile Computing, 6(6):606-620, June 2007.

[2] Aaron Clauset and Nathan Eagle. Persistence and periodicity in a dynamic proximity network. In DIMACS/DyDAn Workshop on Computational Methods for Dynamic Interaction Networks, 2007.

[3] JM Kleinberg. Navigation in a small world. Nature, 406(6798):845, August 2000

[4] Jérémie Leguay, Anders Lindgren, James Scott, Timur Friedman, and Jon Crowcroft. Opportunistic content distribution in an urban setting. In Proceedings of the 2006 SIGCOMM workshop on Challenged networks CHANTS '06, pages 205-212, New York, New York, USA, September 2006. ACM Press.

[5] M. E. J. Newman. The Structure and Function of Complex Networks. SIAM Review, 45(2):167, November 2003.

[6] MEJ Newman. Networks: an introduction. Oxford University Press, 2010.

[7] A.D. Nguyen, Patrick Sénac, Victor Ramiro, and Michel Diaz. STEPS-an approach for human mobility modeling. In NETWORKING 2011, pages 254-265. Springer, 2011. 
[8] John Tang, Mirco Musolesi, Cecilia Mascolo, and Vito Latora. Temporal distance metrics for social network analysis. In Proceedings of the 2nd ACM workshop on Online social networks - WOSN '09, page 31, New York, New York, USA, August 2009. ACM Press.

[9] John Tang, Mirco Musolesi, Cecilia Mascolo, and Vito Latora. Characterising temporal distance and reachability in mobile and online social networks. ACM SIGCOMM Computer Communication Review, 40(1):118-124, January 2010.

[10] D.J. Watts and S.H. Strogatz. Collective dynamics of 'small-world'networks. Nature, 393(6684):440-442, June 1998. 